Ich muß um Ruhe bitten. Unterlassen Sie das, hier werden keine Flugblätter verteilt! Verlassen Sie das Lokal sofort! Können wir die Polizei holen? ( $\mathrm{Zu}$ Dr. Tetzlaff gewandt) Eine Sekunde, wir lassen sie abholen, oder verlassen Sie freiwillig das Lokal? (Tetzlaff bleibt am Rednertisch sitzen. Erregte Zurufe. Ein Polizeibeamter betritt den Saal und führt den Aktionär nach einem kurzen Wortwechsel hinaus. Beifall und Pfiffe.)

Wir fahren jetze in der Tagesordnung fort! (In Richrung eines Flugblattverteilers) Sie haben doch Flugblätter verteilt! - Holen Sie den Mann weg, hinaus mit den Leuten, die sich schlecht benehmen! - Machen Sie, daß Sie rauskommen! (Erneute Zurufe. Ein junger Mann hat ein Spruchband mit der Aufschrift »AEG investiert im Kriegsgebiet!، entrollt. Ein älterer Aktionär versucht, ihm das Transparent wegzunehmen.)

Die Polizei soll hereinkommen! Meine Damen und Herren, ich muß um Silentium bitten! - Ich bitte um Ruhe! - Wer die Versammlung unterbridat, verläßt das Lokal, nehmen Sie das zur Kenntnis! (Die Unruhe hält an)

Ich muß endgültig um Ruhe bitten! Wer die Ruhe stört, wer die Versammlung unterbricht, verläßt das Versammlungslokal! Jetzt also wirklich ohne Rücksichtnahme! (Starker Beifali)

Also, Herr Sörensen, sprechen Sie bitte, etwas Schönes jetzt einmal, etwas Vernünftiges, was wir hören können und hören wollen!

\title{
Allgemeine Geschäftsbedingungen und Justizpraxis
}

Hart macht sich bei seiner Entschleierung der Allgemeinen Geschäftsbedingungen (AGB) als Werkzeug des Monopolkapitalismus unnötige Mühe. ${ }^{1}$ Bei der Durchsetzung der $A G B$ zeigt nämlich unser Wirtschaftssystem sehr offen, wie nah die Verwandtschaft zu einem brutalen Manchesterkapitalismus ist. Darum steht hinter der - mit unzulänglichen Mitteln und mit unzulänglichem Erfolg betriebenen - Abwehr des Bundesgerichtshofs nicht so sehr das Bemühen, »die dem Markt zugeschriebene Funktion durch Korrektur zu realisieren «, als vielmehr, den Konsumenten vor der gröbsten Ausbeutung zu schützen.

$\mathrm{Zu}$ dieser Einsicht konnte Hart nicht kommen, weil er in den Mittelpunkt seiner Untersuchung das Produktionsunternehmen, offensichtlich als Großunternehmen, gestellt hat. Dieses bedient sich aber, wie Mückenberger/Hart an anderer Stelle richtig ausführen², bei Streitigkeiten im Zusammenhang mit der Produktion nicht der staatichen, sondern einer eigenen Gerichtsbarkeit (Schiedsgerichte). Zudem haben sie, mit Ausnahme der Automobilindustric, keinen unmittelbaren Kontakt mit dem Konsumenten.

Für den Konsumenten sind aber die von den Dienstleistungsunternehmen entworfenen AGB wichrig, also die von Banken (Teilzahlungskredite!), Versicherungen, Großversandbetriebe, Reiseunternehmen. Bekannt ist hier ja die vor

\footnotetext{
$1 \mathrm{KJ}$ I971, 269.
}

- KJ I 971,245 . 
allem von den Banken - die ja noch mehr als die Großbetriebe für den deutschen Kapitalismus typisch sind - einsuggerierte Begründung, ihre AGB hätten eine auch im Interesse des Kunden liegende Ordnungsfunktion, weil das BGB keine ausreichende Regelung enthalte. Auch wache ja über ihre AGB - wie auch über die der Versicherungen - eine besondere staatliche Aufsichtsbehörde. Tatsächlich haben aber diese Behörden, wie sich jüngst bei der Kraftahrzeughaftpflichtversicherung gezeigr har, keine ausreichenden Uberprüfungsmöglichkeiten, sie sind auch sactlich und personell mit den zu überprüfenden Organisationen, zumindest mittelbar, verbunden und haben deshalb für diese eine erwünschte Alibifunktion. Kurz: die AGB haben hier dazu geführt, die ohnehin schon kapitalistenfreundliche BGB-Regelung durch ein Diktat der Großbetriebe und Großverbände zu ersetzen. Allerdings sind diese AGB noch geradezu kundenfreundlich, vergleicht man sie mit denen, derer sich in letzter Zeit die Makler bedienen, weshalb es gerade auf dem Gebiet des Maklervertrags immer wieder zu gerichtlidhen Auseinandersetzungen kommt.

Hart kann auch nicht zugestimmt werden, daß die Unternehmen Auseinandersetzungen über die AGB scheuen, zumal sie auch, wie zu zeigen ist, von Musterprozessen keine Nachteile zu befürchten haben. Wohl besteht gegenüber dem Geschäftsfreund eine gewisse Kulanz, gegen den kleinen Mann wird aber in der Regel rüdksichtslos vorgeangen, u. U., wie ich es als Vollstreckungsrichter erlebt habe, wegen einer Restschuld von 4.- DM zum Offenbarungseid geladen. Hierbei ist von Bedeutung, da $B$ in den angeführten Wirtschaftsbereichen die Prozeßinitiative schon längst über Inkassoabteilungen und Vertrauensrechrsanwälten bürokratisch festgelegt ist.

Die Konsumenten dagegen scheuen natürlich den Prozeß. Besonders im Bereich des Hausvertriebs über Abzahlungsgeschäfte. Hier läßt gerade das Vorweisen dichtgedrudkter Formulare durch den Vertreter für die Seriositär des Verkäufers bürgen, die Daumenschrauben in den Bedingungen werden nicht erkannt. Erst reche sind die Kunden hilflos, wenn die Versprechungen des Vertreters nicht eingehalten werden. Symptomatisch war hier ein Strafverfahren wegen Koppelungsgeschäfts. Ein Vertreter hatte Schreibmaschinen verkauft und zugleich für eine andere Firma die Zureilung von Schreibaufträgen zugesagt. Diese hielt die Zusage nicht, die Schreibmaschinenfirma wiederum erklärte, sie habe mit dem Dienstvertrag nichts zu schaffen. Von etwa 80 Geschädigten haben es nur 5 oder 6 zum Zahlungsbefehl kommen lassen; nur einer legte Widerspruch ein, wobei der Amtsrichter gegen ihn entschied - natürlich fehlerhaft -, indem er sich die Argumentation der Schreibmaschinenfirma zu eigen machte.

Eine andere Gruppe der AGB sind nicht gezielter, sondern eher zufälliger Natur, nämlich jene bei kleinen GeschäAtsleuten, Reinigungsbetrieben, Gebrauchtwarenhändler, Kraftfahrzeughandwerker. Diese haben ihre AGB nicht selbst entworfen, sondern sie benützen die ihnen von den Verbänden zugesandten Formulare. Hier haben die Syndici gute Arbeit geleister, d. h. diese AGB sind durchweg die kundenfeindlichsten. Der Gewerbetreibende nimmt die Formulare, um etwas •Schriftliches* abzumachen, versteht in der Regel seine eigenen Bedingungen nicht einmal und nimme auch bei Auseinandersetzungen mit dem Kunden kaum darauf Bezug. Wirksam werden sie erst, wenn er einen Rechtsanwalt einschaltet, der schnell erkennt, welche siegreiche Waffe sein Mandant in den AGB besitzt.

Die AGB wirken sich deshalb besonders verbängnisvoll aus, weil der Durchschnitrsrichter ihnen unkritisch gegenüber steht. Hier befinden wir uns in einem sehr komplexen Bereich richterlichen Verhaltens. Von seinem Vorverständnis aus steht der Durchschnittsrichter aufgrund seiner wirtschaftsfremden Herkunft und 
Ausbildung der Betätigung der Wirtschaft im Rechtswesen mit Skepsis, ja, wie Wiethölter betont, mit Ressentiment gegenüber. Hinzu kommt, daß sich ein Richter wie jeder andere Kunde in der Regel nicht die AGB auf dem Annahmeschein durchliest, bevor er seinen Anzug zum Reinigen, sein Kraftfahrzeug zur Reparatur bringt. Wird er aber mit einem AGB-Prozeß befaßt, so hält er sich doch wieder an das dogmatische Gerüst des Systems, an die Privatautonomie. Zudem entspricht der Implikation durch die Ausbildung die Interpretation von Normen, die ja bei den AGB durch die Verbandssyndici ngesetzesreif « formuliert werden. Der Richter erspart dabei die Vernehmung von Zeugen und Parteien, die ja im Zivilprozeß als eine Tätigkeit minderen Ranges angesehen wird. Sein Gewissen beruhigt der Richter mit der Erwägung, der Kunde solle gefälligst lesen, was er unterschreibe (obgleich der Richter als Kunde nicht anders verfahren wird).

Komplex ist weiter, daß dagegen der Bundesgerichtshof, der ansonsten als Nachfolger des Reichsgerichts die Unternehmerinteressen begünstigt, bei den AGB verbraucherfreundlich ist. Komplex ist endlich, daB die sich immer stärker am Bundesgerichtshof ausrichtenden Instanzgerichte ihm im Bereich der AGB weitgehend die Gefolgschaft versagen. Dies mag zum Teil darauf zurückzuführen sein, daß nur ein kleiner Teil der AGB-Prozesse wegen der Streitwerthöhe in die Revisionsinstanz geht, so in Kredit-, Makler- und Bausachen. ${ }^{3}$ Aber selbst in diesem Bereich - bis auf die Abzahlungsgeschäfte, bei denen eindeutige gesetzliche Intentionen vorliegen - vermag sich der Bundesgerichtshof nicht gegenüber den Instanzgerichten durchsetzen. Der Grund liegr aber nicht in unterschiedlichen Rechtsauffassungen, sondern in dem - schuldlosen - Unvermögen der Instanzgerichte, die Informationsflut zu bewältigen: eine dreistellige Zahl von AGB-Entscheidungen des Bundesgerichtshofs, überquellende Kommentarbelege, Schwierigkeiten bei der synoptischen Prüfung, ob die in einer Bundesgerichtshofsentscheidung untersuchte $A G B$ sich mit derjenigen vergleichen läßt, um die es im konkreten Fall geht; Hilfslosigkeit der Richter im Hinblidk auf die zu leistende wirtschafts- und gesellschaftpolitische Argumentation.

Endlich lähmt den Richter die Vergeblichkeit seines Bemühens. Ist nämlich in letzter Instanz eine AGB-Klausel für nichtig erklärt worden, so hat binnen 8 Tagen das Unternehmen eine leicht abgeänderte Fassung für seinen Geschäftsbetrieb vorliegen, und es wird 8 Jahre dauern, bis in letzter Instanz entschieden ist, daß auch diese Fassung nicht vor dem Recht bestehen kann. Selbst wenn die Richter die Gefahren der Rechtsverschmutzung durch die AGB erkannt hätten - was leider nur für wenige zutrifft -, so reicht ihr Instrumentarium zur Abwehr genauso wenig aus wie dasjenige der Gesundheits- und Gewerbeämter zur Abwehr der Umweltverschmutzung. Kein übertriebener Vergleich! Selbst der Altmeister der neueren juristischen Rechtstheorie, Esser spricht im Hinblids auf die AGB von einer "denaturierenden privaten Praxis«. ${ }^{4}$

Wer zeigen will, wie sehr unser Rechts- und Gerichtssystem dem Kapitalismus dient, der braucht keine subtilen Hinterfragungsanalysen zu machen; es genügt schon eine empirische Untersuchung von AGB-Prozessen, und die erschreckende Maßlosigkeit des Kapitalismus im Rechtswesen wird sichtbar.

Theo Rasehorn

${ }^{3} \mathrm{AGB}$ im Bauwesen stehen gewissermaßen zwischen den beiden aufgezeigten Gruppen, d. h. es gibt Unternehmen, die gezıeit damit arbeiten und wieder andere, bei denen sie, wie angezeigt, mehr zufällig in Erscheinung treten.

4 Vorverständnis und Methodenwahl in der Rechtsfindung, Frankfurt 1970, S. I 50. 